\title{
The use of exploratory procedures by blind and sighted adults and children
}

\author{
Ans Withagen • Astrid M. L. Kappers • Mathijs P. J. Vervloed • \\ Harry Knoors • Ludo Verhoeven
}

Published online: 12 June 2013

(C) Psychonomic Society, Inc. 2013

\begin{abstract}
The study examined exploratory procedures (EPs) of congenitally blind and sighted children and adults on a haptic match-to-sample task. The aim was to examine the influence of age, visual status, and familiarity on the use of EPs when people haptically examine the object properties of weight, size, exact shape, and texture. EPs in the first and last of four series of trials were compared. The results showed that all four groups chose the same dominant EP for examining the four different object properties, all of them in agreement with the ones found by Lederman and Klatzky (Cognitive Psychology 19:342-368, 1987). Children were found to use more EPs, rather than using only the most efficient EP, for the dimension under study. Overall, performance was affected more by age than by visual status, and repeating the task led to increased efficiency in all groups. To describe exploratory behaviors in more detail, actions were introduced. Actions are single or sequential hand movements occurring in parallel with the EPs or apart from the EPs. The use of actions explained, in part, individual variation among the participants.
\end{abstract}

\footnotetext{
A. Withagen $(\bowtie)$

Royal Dutch Visio, Centre of expertise for blind and partially sighted people, Oud Bussummerweg 76, 1272 RX Huizen, The Netherlands e-mail: answithagen@visio.org

A. M. L. Kappers

VU University Amsterdam, Faculty of Human Movement Sciences, Amsterdam, The Netherlands

M. P. J. Vervloed $\cdot$ H. Knoors $\cdot$ L. Verhoeven Behavioural Science Institute, School of Educational Science, Radboud University Nijmegen, Nijmegen, The Netherlands

H. Knoors

Royal Kentalis, Sint Michielsgestel, The Netherlands
}

Keywords Blindness · Touch · Exploratory procedures · Child $\cdot$ Object properties

\section{Introduction}

Given the overt nature of tactual exploration, mental processes and cognitive strategies can be deduced more easily from touch than from the other senses. Typically, this is done by looking at exploratory procedures (EPs), first described by Lederman and Klatzky (1987) as certain types of hand movements made to get specific information about object properties. Typical patterns emerge once people are asked about object properties, such as weight, volume, or shape. For instance, the typical pattern for exploring the dimension of exact shape is contour-following (CF), a dynamic procedure in which one or more fingers trace the contours of an object.

In view of our clinical interest in blind children and adults, we wondered whether blind people use the same EPs to extract information from objects. In a previous article (Withagen, Kappers, Vervloed, Knoors, \& Verhoeven, 2012), we compared the performance of four groups (blind adults, sighted adults, blind children, and sighted children) with regard to accuracy and response times in a match-tosample task with different object dimensions (shape, weight, texture, and volume). The experimental task was performed 4 times to investigate the effect of familiarization and practice. We showed that, with regard to accuracy and response times on the four different dimensions, age was more important than visual status. The differences between children and adults were especially large for exact shape, which turned out to be significantly more difficult for children than for adults. For accuracy, the effects of practice were 
significant only for perceiving texture. In contrast, repeating the experiment had a significant effect on all response times; all groups became faster over the series. There were no significant differences in performance between blind and sighted children. The blind adults were faster than the sighted adults in response times, especially for exact shape, without decreasing in the level of accuracy. During this previous study, hand movements were recorded (but not yet analyzed). The main objective of the present study is to investigate whether the differences between the groups were caused by the use of different exploratory strategies by blind and sighted adults and children.

Lederman and Klatzky (1987) studied the hand movements in a mutually exclusive scoring system and chose to score the most prominent EP in the case where two EPs occurred together. In a later study, Klatzky and Lederman (1993) made predictions about the compatibility of two EPs occurring together. To actually study this compatibility, the option of scoring simultaneous EPs was added to the present study.

\section{Manual exploration in sighted children}

Previous research has shown that the use of EPs is age related. Bushnell and Boudreau (1991) noted that the order in which haptic perception of various object dimensions initially develops in infants is determined mainly by the temporal sequence in which the associated EPs can be performed, which, in turn, appears to depend on the level of motor development. Morange-Majoux, Cougnot, and Bloch (1997) confirmed that as early as the age of 4 months, infants are capable of discriminating coarse variations in texture. The hand movements proved similar to the adult EP of lateral motion (LM). At the same age, infants use their whole hands to explore objects, and by the age of 6 months, they start using their fingertips. Bushnell and Boudreau suggested that in order to perceive particular properties of an object, an infant must be able to perform the requisite hand movements. For example, to determine the texture of an object, a child needs to be capable of rubbing a finger across a surface. It is only when the infant has developed more sophisticated exploratory strategies, such as using one hand to hold the object and the other to explore, that he or she may be able to determine the shape of an object. According to Bushnell and Boudreau, the onset of all EPs is thus in the first year of life.

Kalagher and Jones (2011) recently assessed 36 sighted children (3-5 years) and 20 adults with a haptic match-tosample task. The participants could not see their hands during the experiment. They received objects and were instructed to compare the sample objects with the test objects with respect to the properties shape, texture, rigidity, and weight. There was always one object that was identical to the sample object. Both accuracy and hand movements of the children were studied and compared with the performance of adults. Kalagher and Jones concluded that these young children behaved in the same way as adults. To solve the tasks, they produced adult-like EPs. Children applied pressure to match on rigidity, CF for shape, LM for texture, and unsupported holding (UH) for weight. The results indicated that even 3-year-olds chose the most adequate EPs to gather specific perceptual information. Moreover, children of all age groups were able to match the objects for all dimensions at near ceiling levels. Klatzky, Lederman, and Mankinen (2005) carried out a study on the use of EPs by sighted children and focused on whether preschool children (mean age 4.5 years) could make appropriate judgments about tool functions by using either vision or vision and touch. The children proved to be sensitive to the characteristics of the tool and to the constraints on its functions. They used vision to explore the size of the tool and the EP of pressure (PR) to learn about the rigidity of an object. Berger and Hatwell $(1993,1995,1996)$ found that even 5-year-old children can classify objects on the haptic dimension of texture by using the EP of LM. The EPs to apprehend size (enclosure [EN], CF) were scarcely used to classify objects. In contrast, Schwarzer, Küfer, and Wilkening (1999) did not find a preference for classification based solely on texturethat is, by the use of the EP of LM. They found that children tended to use more hand movements besides LM, such as $\mathrm{EN}$ and rotation, to explore and classify the dimension of texture. Even 4-year-old children used these procedures frequently. Also, the EP of CF was already observed at this age.

\section{Hand movements in blind children}

There are only a few studies on the development of EPs in blind children. Landau (1991) examined haptic object exploration in three congenitally blind infants between 18 and 36 months of age. In her study, blind infants used scratching to explore textures and rotation to explore the shapes of objects. Schellingerhout, Smitsman, and Van Galen (1997) examined the EPs that eight blind infants between 8 and 24 months of age used to explore textured surfaces. The children received different gradient textures on a table in front of them while seated on their mothers' lap. The children explored the surfaces spontaneously and without any instructions. Schellingerhout et al. saw that the infants used a wide range of exploratory strategies, such as touching, hitting, fingering, rubbing, and mouthing, and that there were age-related differences in the use of exploratory strategies. The youngest children showed limited use of fingering and a rudimentary form of rubbing, whereas the oldest 
did not show oral exploration anymore. The exploratory behaviors varied depending on the textures the children explored.

According to Millar (2008), young congenitally blind children often perform significantly poorer on unfamiliar spatial tasks than do blindfolded sighted children. She noticed that the differences between the two groups depended on the nature of the task and the kind of information that was available to the children. Millar (1974) performed a haptic match-to-sample recognition task, using small nonsense shapes with blind and sighted children. The blind children were faster responders but were somewhat less accurate. On the basis of her experiments, Millar (1994) concluded that the need for informational redundancy is necessary for blind children to get the same experiences and draw the same conclusions as sighted children. She suggested that the convergence and overlap of tactual inputs provide the redundancy needed to interpret information about objects and their spatial positions.

\section{Blind and sighted adults}

Davidson (1972) and Davidson and Whitson (1974) compared the exploratory behavior of blindfolded sighted and blind adult observers when haptically evaluating the curvature of a stimulus, which was either convex or concave. They concluded that the blind participants were more efficient in their haptic strategies, because they used the whole hand, whereas the sighted participants used only two fingers. However, after a training in which the sighted participants were forced to use the same mode of exploration as the blind participants, the two groups produced similar results. Davidson and Whitson concluded that with the use of the adequate haptic strategy, sighted people can achieve the same level of accuracy as the blind in this haptic task.

Heller (1989) also studied the influence of vision and visual imagery on the perception of textures. His results showed that for texture, there were no differences in performance between blind and sighted adults. According to Heller, visual imagery may not be needed for substancerelated object qualities - that is, material properties such as texture, hardness, thermal properties, and weight-whereas visual imagery may be helpful for judgments about geometrical properties such as shape. Grant, Thiagarajah, and Sathian (2000) studied whether the blind are superior, in comparison with the sighted, in their tactile sensitivity. Initially, the blind outperformed the sighted on a hyperacuity task where the participants were allowed to use active touch. However, after practice, both groups performed at the same level. They concluded that people deprived of vision do not develop supernormal touch sensitivity but, rather, learn to use it more proficiently. Goldreich and Kanics
(2003) also studied tactile sensitivity of blind and sighted people, but now the participants were only allowed to make use of passive touch. It turned out that tactile acuity of the blind participants was significantly better than that of the sighted participants. So it seems that tactual acuity is better in blind versus sighted people when passive touch is used but that, after practice and with use of active touch, both groups perform equally.

Several studies (Cornoldi, Fastame, \& Vecchi, 2003; Cornoldi \& Vecchi, 2000; Vanlierde, \& Wanet-Defalque, 2004; Vecchi, Tinti, \& Cornoldi, 2004) showed that when objects are presented to blind persons, congenital absence of visual perception does not prevent one from processing mental images. Spatial images are probably organized differently in blind persons, in comparison with sighted persons, because they are derived from tactile and spatial information sources. These kinds of mental representations can be used by blind persons in tactile-spatial tasks. However, increased complexity of the task (e.g., when people have to recall more than a single spatial pattern) has a negative influence on the performance of blind people.

Recently, Rovira, Deschamps, and Baena-Gomez (2011) let blind and sighted adolescents perform two tasks in which two-dimensional geometrical shapes with raised patterns had to be rotated mentally. They found that the blind participants performed more effectively, which was tentatively attributed to the fact that they used multifingered and bimanual exploration during the experimental task, whereas the sighted adolescents tended to prefer to use only one hand during exploration of the objects. Furthermore, they used twice the time to explore the objects and give their responses, in comparison with the blind adolescents.

\section{Aim of the study}

In previous research, it has been found that the performance on tactual exploration tasks may vary as a result of the dimensions being studied, practice with the task, and the age and visual status of participants. However, in the studies conducted so far, no attempt has been made to examine the initial and repeated exploration behavior on multiple dimensions as a function of age and visual status in one and the same design. Therefore, the present study focused on the repeated tactual exploration of blind and sighted adults and children. The goal of the study was to gather both quantitative and qualitative information about the haptic strategies used by these groups. The research question was twofold: (1) Do blind and sighted adults and children choose the same EPs in tasks concerning exact shape, weight, volume, and texture, and (2) what is the effect of practice? The effect of practice on the use of EPs was studied by comparing the 
first and last series of trials. Since no previous studies are known that have studied the effect of practice, we investigated whether the EPs change over time (for instance, more simultaneous use of different EPs or increased use of the most efficient EP to solve a specific task). We wanted to see whether an effect, either a learning or a fatigue effect, would arise after repeating the experiment 3 times. In addition, we were also interested in the possible differences between children and adult and between blind and sighted in the use of EPs. On the basis of Millar's (1997) CAPIN theory, we expected that children and blind people would show more redundant behaviors during the experiment but also that they would profit more from rehearsals than would sighted adults. To observe the hand movements in more detail, actions were defined and scored in addition to the EPs. Actions are extra hand movements, without distinct onand offset times, that cannot be scored as an EP and that participants execute to gather extra information about an object.

\section{Method}

\section{Participants}

Sixty-one participants, 31 adults and 30 children, took part in the experiment. Each participant belonged to one of four groups:

1. congenitally blind adults $(n=16$; mean age $=38.8$, $S D=8.1 ; 8$ females, 8 males $)$

2. sighted adults $(n=15$; mean age $=39.4, S D=10.7$; 8 females, 7 males)

3. congenitally blind children $(n=15$; mean age $=9.3$, $S D=1.7 ; 7$ girls, 8 boys)

4. sighted children $(n=15$; mean age $=9.0, S D=1.5 ; 7$ girls, 8 boys).

Within the age groups, the blind and sighted participants were matched on age, gender, and level of education. We wanted to reduce the chance that children did not understand the concepts of the dimensions under study and, therefore, enrolled children between 7 and 12 years of age (mean age 9 years) in the study. By excluding younger children, we hoped to diminish the number of children in the preoperational phase of cognitive development. The rather large age range was necessary to enroll a sufficient number of participants in the study. The blind children attended mainstream schools in the Netherlands and Flanders and were matched with sighted classmates. The blind adults were recruited from the social network of the first author and a Web site for the blind and visually impaired in the Netherlands. Informed and written consent was received from all adult participants and the parents of the children. The total experiment, including practice trials, took about $1.5-2.5 \mathrm{~h}$. For the children, there was always a short break between series 2 and 3, in which they got a drink. All adults performed the experiment without a break. The children were tested at their own school, whereas the adults were assessed either at home or at work.

\section{Materials}

Four out of the nine original object sets in the study by Lederman and Klatzky (1987) - namely, texture, weight, volume, and exact shape - were replicated with the permission and help of the authors. All objects were unfamiliar, meaningless, and functionless objects and, therefore, were difficult to label (see Fig. 1). The objects involved in this experiment had a size of $4-12 \mathrm{~cm}$; they were too big to enclose in one hand, especially for the children. Within each set, the objects differed on the dimension under study and an additional dimension with distracting information for the target dimension. This additional dimension was the size of the objects in exact shape, the material and size in weight, and the shape of the objects in both texture and volume.

For each object dimension, there were 16 threedimensional-stimuli, divided into four sets. Each set consisted of 1 standard object and 3 comparison objects, of which 1 was the best, but not an identical, match to the standard. In total, there were 64 objects (see Fig. 1). An additional practice set was developed for instruction and familiarization. A more detailed description of the object sets can be found in Withagen et al. (2012).

Procedure and setting

The procedure was a slightly adapted version from the study of Lederman and Klatzky (1987) and is also described in Withagen et al. (2012). For practical reasons, four, and not nine, dimensions were studied. Participants were not blindfolded, but their view of the objects and their hands was blocked by a curtain. This was done because a pilot study showed that most children were bothered by being blindfolded. The experiment was repeated 3 times in order to study the effects of practice and fatigue.

Participants sat at a table opposite the experimenter. The experimenter and the sighted participants could see each other over the curtain that hung over a stage and that blocked their view of their hands. The participants put their hands under the curtain at the side of the experimenter, where they received the objects. Each trial started when the participants laid the back of their hands down on the table, palms upward. Exploration started when the participants received the object in their hand(s). To indicate the 
Fig. 1 Object sets for the dimensions of texture (a), weight (b), volume (c), and exact shape (d). The standard objects are shown in the first column, followed by the comparison objects in the same row; the second object in each row is the best match

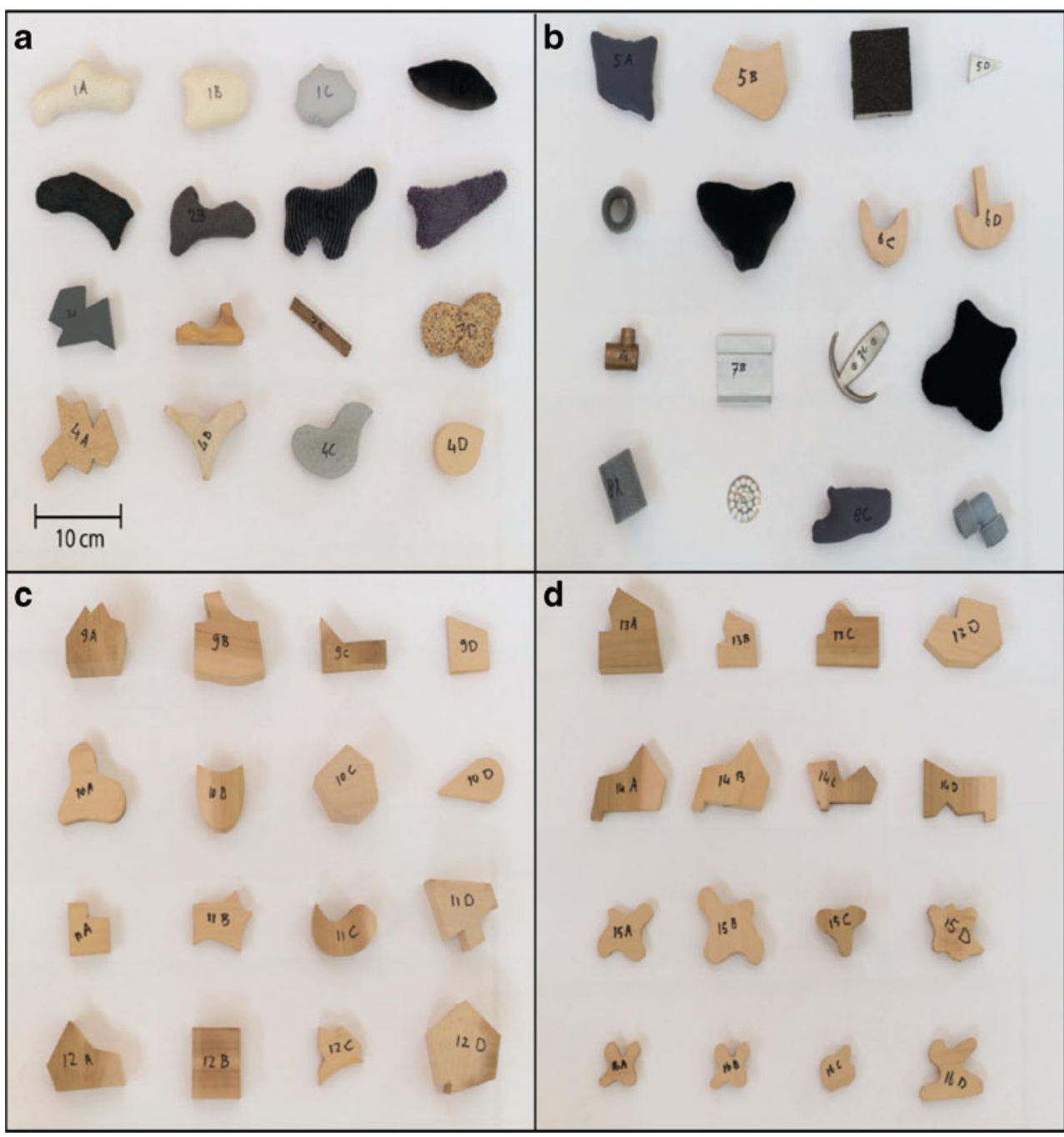

end of a trial, the participants had to lay both hands back on the table, palms up, fingers stretched.

The experimenter first presented the standard object, which the participants were asked to explore for one of four dimensions: texture, weight, volume, or exact shape. Next, the three comparison stimuli were given one after the other, and the participants were asked which of the three matched the standard best with regard to the required dimension. The participants were told that there would be no identical match to the standard. Simultaneous comparison of the stimuli was not possible, and the participants were allowed to explore the objects only once. There was no time limit for responding. The participants were instructed with the following descriptions for the different dimensions. For the dimension of exact shape, the question was the following: "What is the exact outline of a shape? Ignore the size of the object, but concentrate only on the outline. Which object has the same outline as the standard, although it might be larger, thicker or smaller?" Texture was questioned as follows: "How does the object feel on the surface. Do not pay attention to the shape or size, but concentrate on the surface. How does it feel, is it, for instance, soft or rough?" The instruction for the dimension of volume was the following: "What is the size of the object? Ignore the shape, but explore the size at all the sides of the object." If the participant did not understand the instruction, extra information was given: "Imagine you can make a hole in the object and fill it with water. In which object would fit about as much water as in the standard? Would it be number 1, 2 or 3?" For the dimension of weight, participants were asked the following: "What is the weight of the object in your hands; does it feel heavy or light? Compare the weight of the standard with the other objects."

Every assessment started with practice trials that were intended to instruct the participants what the dimensions meant and to make them familiar with the match-to-sample task. During the practice trials, the participants received two trials with objects, not in the actual object-set, to practice the procedure. Furthermore, it was checked whether the participants understood the concepts of the dimensions they were asked to match. Immediately after the practice trials, the experiment started with four trials for each of the four sets of dimensions; the same instruction for the dimensions was 
given, as described above. Each participant received the four object sets in a randomized sequence, for a total of 64 objects. In the original study of Lederman and Klatzky (1987), the experiment finished after all sets were explored. Since we were interested in the influence of familiarity and learning, we repeated the experiment 3 times (all in one assessment). In total, there were four series with the four object-sets, bringing the total number of objects to be explored to 256 ( 4 series $\times 4$ dimensions $\times 4$ trials $\times 4$ objects). All trials were recorded on video by a camera placed on a tripod behind the experimenter. Practice trials were not recorded.

\section{Hand movements}

The hand movements were divided into two categories, exploratory procedures and actions. Some manual behaviors could not be scored as EPs and were, therefore, called actions. Actions are extra hand movements that are mostly constellations of hand motions that occur sometimes in parallel with the EPs and, in other cases, apart from the EPs. Some actions convey some obvious intentions of the participants, such as estimating size or global perception of shape; others consist of sequences of behaviors that are not captured by EPs (see Table 2). All hand movements were analyzed with the Observer XT software from Noldus (Noldus Information Technology, 2008). The scoring rules for the EPs were based on the original scoring rules of Lederman and Klatzky (1987). A difference from the original study is that we embedded the possibility of scoring EPs simultaneously. Both frequency and duration were scored for EPs. Only frequency was scored for actions, since actions either lasted for very brief periods or were sequences of different hand movements in which EPs sometimes also occurred.

Besides the EPs of LM, UH, EN, CF, and PR, we scored task maintenance (TM) and unclear (UC). Under TM, all hand movements used for the benefit of the performance of an EP were scored. The observers used UC when the recordings were unclear or invisible for them. Lists of EPs and actions are shown in Tables 1 and 2, respectively.
Five students, naive to the experimental goals, were trained in scoring by observing the tapes of a pilot study, until sufficient agreement was reached (i.e., Cohen's kappa coefficient above .80). The interrater reliability was determined by scoring the tapes of 10 participants, each by five different raters. Tapes were randomly selected from the beginning, midpoint, and end of the study. Reliability was determined by calculating the intraclass correlation coefficient, which is also presented in Tables 1 and 2.

\section{Analyses}

Response time was defined as the duration of the total exploration. Each exploration started when the participants received the first object in their hands; the exploration ended when the participants stopped exploring the last comparison object (i.e., haptic exploring; not when the participants gave the response to the question). The durations of the EPs per participant were calculated per dimension. Because of unequal response times between participants, relative scores were calculated by converting the durations into a percentage of time a participant executed a specific EP. Sometimes, EPs occurred simultaneously. Analyses of the data showed that the EP predicted by Lederman and Klatzky (1987) to solve a task best was always involved when two EPs occurred simultaneously. For this reason, the occurrence of combined use of EPs with a specific dimension was indicated by adding the plus sign to the most prominent EP. Periods in which participants were not using EPs were scored as the category rest. During these periods, participants either could make hand movements used for the benefit of EPs (TM) actions or showed undefined or unidentifiable behaviors (UC).

For ease of presentation, only the results of the analyses of the first and last series of trials will be presented. The mean number of actions was calculated by dividing the total number of occurrences of each event by the number of participants.

Table 1 Exploratory procedures: descriptions and intraclass correlations

\begin{tabular}{llr}
\hline Exploratory procedures & Description & Intraclass correlation \\
\hline Lateral motion & The fingers are rubbed sideways on the surface of an object. & .93 \\
Unsupported holding & $\begin{array}{c}\text { An object is lifted away from any supporting surface and is maintained in the } \\
\text { hand without any effort to mould the hand to the object. } \\
\text { Both hands are put around the outer surface of an object. }\end{array}$ & .99 \\
Enclosure & $\begin{array}{l}\text { This is a dynamic procedure in which one or more fingers } \\
\text { trace the contours of an object. } \\
\text { Contour following }\end{array}$ & $\begin{array}{l}\text { Pressure is applied to a part of the object- for instance, the } \\
\text { object is poked or pinched. }\end{array}$ \\
Pressure & & .97 \\
\end{tabular}


Table 2 Actions: descriptions and intraclass correlations

\begin{tabular}{|c|c|c|}
\hline Actions & Description & $\begin{array}{l}\text { Intraclass } \\
\text { correlation }\end{array}$ \\
\hline Throw over & $\begin{array}{l}\text { Throw the object from one hand into the other; it is often a very smooth } \\
\text { operation and is repeated several times. }\end{array}$ & 1.00 \\
\hline Pick up and Drop & Participant picks up the object with one hand and lets it drop on the other hand. & 1.00 \\
\hline Brief touch & $\begin{array}{l}\text { The object is lying on the extended hand, most of the time in the position of UH; } \\
\text { the participant only touches the object softly to get a bit of information (quick glance) } \\
\text { about the object and material. This event is not scored if the participant is really exploring } \\
\text { or pressing on the object }\end{array}$ & .92 \\
\hline Examine features & The participant is exploring features, like a hole in a ring (put finger through). & .99 \\
\hline Estimating size & $\begin{array}{l}\text { Specific movements are made by hands or fingers with the goal of measuring size or distance. } \\
\text { Typical is the folding of two hands together over the object and giving extra pressure to } \\
\text { get more information about size. Sometimes the fingers or palms of the hand play the role of a ruler; } \\
\text { people measure the length of an object with one or more fingers. }\end{array}$ & .95 \\
\hline $\begin{array}{l}\text { Global perception } \\
\text { of shape }\end{array}$ & $\begin{array}{l}\text { The participant places the fingers of one hand at the outline of an object, to get an indication about the } \\
\text { shape of an object. It differs from contour following in that the contour of the object is not traced. }\end{array}$ & .98 \\
\hline
\end{tabular}

To integrate accuracy scores and response times, mean efficiency scores were calculated per group by averaging the ratios of accuracy and response time of all individual participants. To analyze the results on the efficiency scores, an age (adults-children) $\times$ visual status (blind-sighted $) \times$ series (2) general linear model (GLM) analysis was performed. Age and visual status were treated as between-subjects and series as the within-subjects variables. After this analysis, analyses for both age groups separately were conducted, with visual status as the between-subjects variable.

\section{Results}

Exploratory procedures per dimension

Figure 2 shows the relative duration of EPs for the four different groups on series 1 and 4 for the dimension of exact shape. It can be seen that the most important EP to gather information about the exact shape of an object is CF. In series 1 , all four groups of participants executed CF more than $80 \%$ of the time during which they were exploring the object. Apart from the sighted adults, who in series 4 still showed a high level of CF ( $>95 \%$ ), the other three groups showed a shift in their exploratory behavior. EN was added as a second EP, especially by the blind children, or in combinations of EPs $(\mathrm{CF}+)$.

For all four groups, with the dimension of weight, the most commonly used EP was UH in both series 1 and 4 (see Fig. 3). Especially, the children also used other EPs. In series 1, besides UH, the EP of EN was used frequently. Note, however, that in series 1, the category rest was also quite high, which means that the participants executed other hand movements than just EPs. In series 4, the adults still executed UH for about $80 \%$ of the time to gather information about weight. Both groups of children performed UH less than did the adults.

Since we could not judge from Fig. 3 whether all children performed in the same manner with regard to the dominant $\mathrm{EP}$ - that is, $\mathrm{UH}$ - we looked at the distribution of individual scores for this EP (see Fig. 4). Most adults used the EP of UH for a considerable amount of time during exploration of the dimension of weight. This can be seen in Fig. 4 by the skewness to the left of the distribution of the adults. The exploratory behaviors of both groups of adults resembled each other. However, within both groups of children, there was more variation in exploratory behaviors than in the adult groups. This meant that the low score on the dominant EP of UH for the groups of children (see Fig. 3) was caused by a large variation in the percentage of use of $\mathrm{UH}$; some children hardly used this EP, whereas a few children behaved like adults - that is, showed UH a large proportion of the time.

For the dimension of volume, shown in Fig. 5, the EP of EN was performed most by all four groups. The next most used EP was CF. In series 4, there was a small increase visible in the use of combination of EPs - that is, $\mathrm{EN}+$.

In Fig. 6, the proportion of EPs for the dimension of texture is shown. LM was the dominant EP for all four groups. However, the EPs of PR and EN were also performed regularly in this task, especially by both groups of children. Since we were interested in how variable the use of the dominant EP of LM was for the two groups of children, we looked again at the distribution of individual scores. The distribution of the scores resembled that in Fig. 4; there were large differences between the children in how often they used the EP of LM, from scarcely to very often. Adults behaved more similarly to each other; that is, most of the times, they executed LM. 
Fig. 2 Percentage of time exploratory procedures were used with the dimension of exact shape for the four groups for series 1 (a) and series 4 (b). $\mathrm{CF}=$ contour following, $\mathrm{EN}=$ enclosure, $\mathrm{LM}=$ lateral motion, $\mathrm{UH}=$ unsupported holding, $\mathrm{PR}=$ pressure
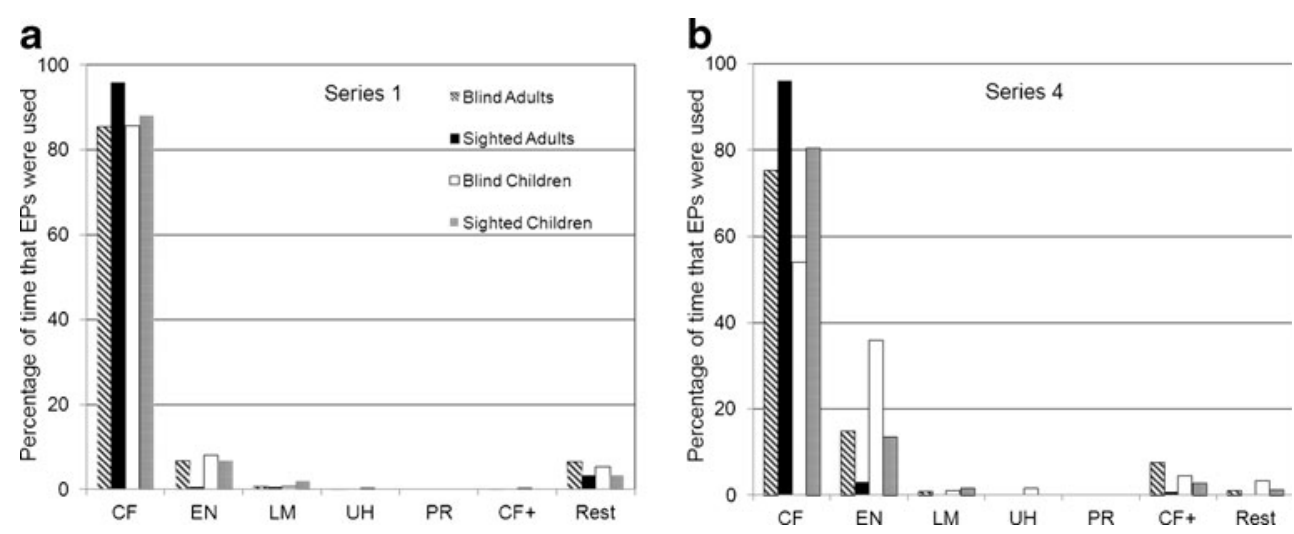

Actions per dimension

Figure 7 shows the mean number of actions per group performed during exploration. Most actions occurred during the tasks of exact shape and weight. The event of brief touch, where the participants got only a quick glance of the object, was performed for all the dimensions and by all four groups of participants, although not with the same frequency. The actions of throw over, pick up and drop, and examine features seemed to belong to the dimension of weight and were not observed on the other dimensions. The event of throw over was executed most of the time by the adults, and the event of examine features seemed to be preferred more by the children. The event of global perception was almost performed only in relation to the dimension of exact shape; in series 4, the frequency of this event increased, especially for the adult groups.

Comparisons with the data of Lederman and Klatzky

Table 3 compares the data from Lederman and Klatzky's 1987 study and the present study and shows the mean duration of the EPs after each question to match the sample on one of the four dimensions. The data for the sighted adults in the present study bore a close resemblance to the data of Lederman and Klatzky. This was confirmed by a significant and very high correlation for the mean duration

Fig. 3 Percentage of time exploratory procedures were used with the dimension of weight for the four groups for series 1 (a) and series 4 (b). $\mathrm{CF}=$ contour following, $\mathrm{EN}=$ enclosure, $\mathrm{LM}=$ lateral motion, $\mathrm{UH}=$ unsupported holding, $\mathrm{PR}=$ pressure
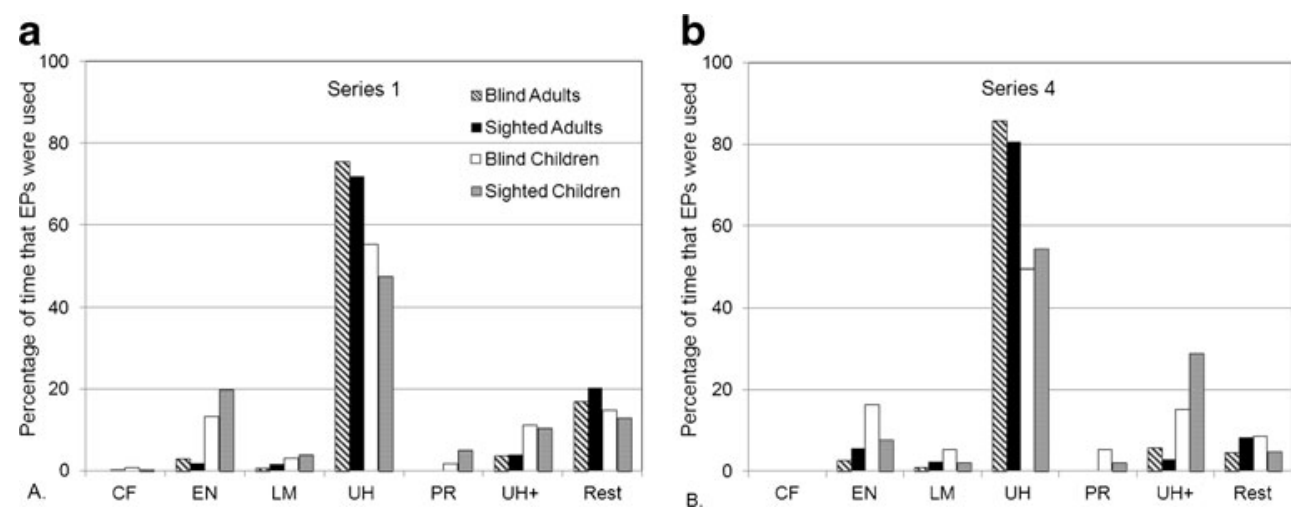

of EPs per object on each dimension between the students in the Lederman and Klatzky study and the sighted adults in the present study $(r=.98, \mathrm{p}<.01)$. Moreover, the correlations were also high between Lederman and Klatzky's participants and the other three groups in the present study ( $r=.92$ for sighted children, $r=.91$ for blind adults, and $r=.86$ for blind children, all $p \mathrm{~s}<.01)$.

\section{Efficiency scores}

To study the overall impact of EPs and actions on accuracy and response times, for each individual an efficiency score was calculated by dividing accuracy by response time, irrespective which EPs or actions were used (see Table 4). The higher the score, the more efficient the person used his or her tactual skills.

For the four dimensions, separate GLMs were performed on the efficiency scores, with age (adults, children) and visual status (sighted, blind) as between-subjects factors and series (1, 2, 3, and 4) as the within-subjects factor. For clarity and consistency with other tables and figures, only series 1 and 4 are shown in Table 4 . For exact shape, there was a main effect for visual status, $F(1,57)=12.334$, $p=.001, \eta_{p}{ }^{2}=.178$. The blind participants were more efficient than the sighted participants. There was also a main effect for series $F(3,171)=8.815, p<.001, \eta_{p}{ }^{2}=.134$. Efficiency increased over the series. Analyses for the blind 
Fig. 4 Number of participants who used the exploratory procedure (EP) of unsupported holding (UH) for a certain percentage of time in series 4 with the dimension of weight for the different groups of participants: a blind adults $(n=16), \mathbf{b}$ sighted adults $(n=15)$, c blind children $(n=15)$, and $\mathbf{d}$ sighted children $(n=15)$ a

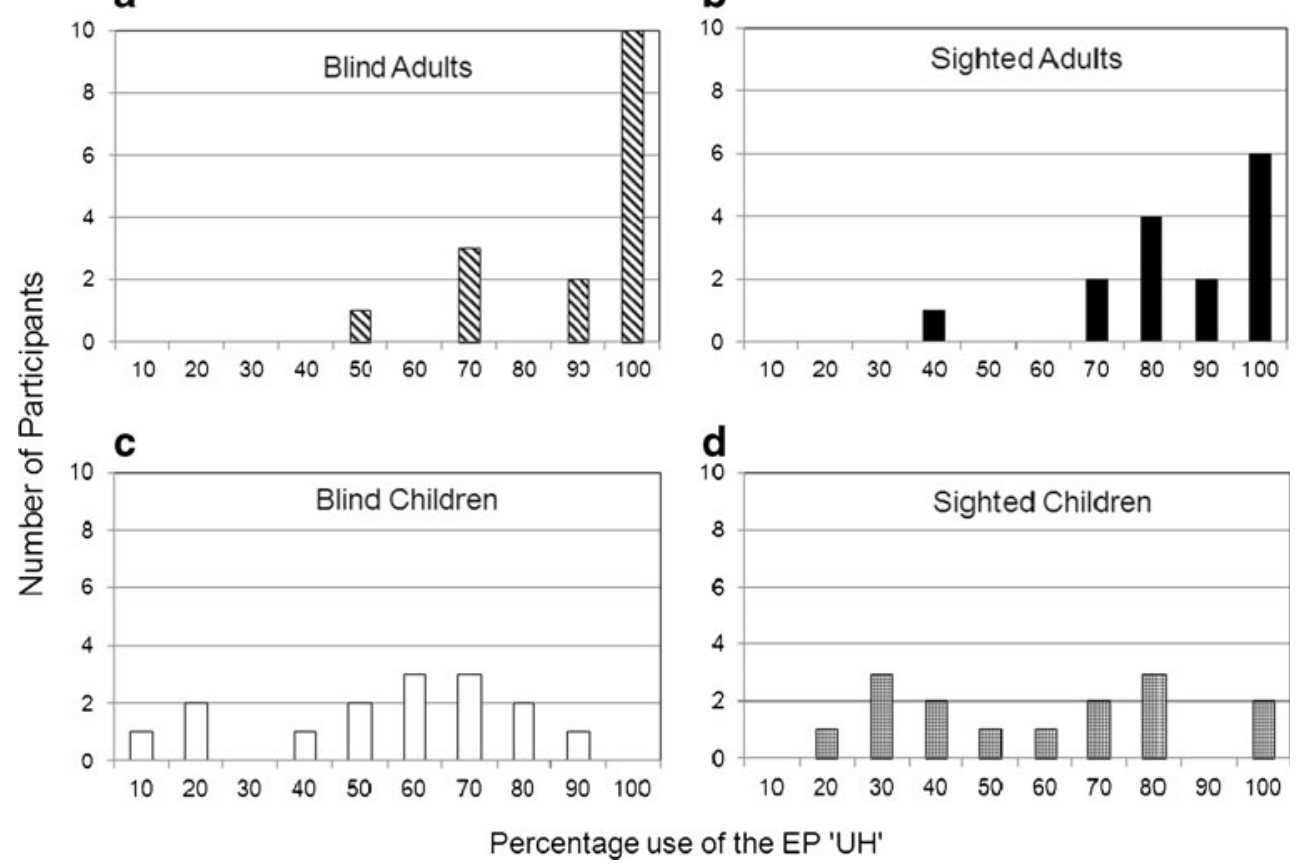

and sighted groups separately showed a main effect for series for both groups. The blind participants, $F(3,87)=$ 5.194, $p=.002, \eta_{p}{ }^{2}=.152$, as well as the sighted participants, $F(3,84)=4.783, p=.004, \eta_{p}{ }^{2}=.146$, became more efficient over time. There was no main or interaction effect for age.

For the dimension of weight, there was an interaction effect of age with the repeated measurement of series, $F(3,171)=4.236, p<.006, \eta_{p}{ }^{2}=.069$. There was also a significant main effect for age, $F(3,171)=3.797, p=.011$, $\eta_{p}{ }^{2}=.062$, and repeated measurement of series, $F(1,57)=$ $15.553, p<.001, \eta_{p}{ }^{2}=.214$. Separate analyses per age group showed that these significant results were caused by the children. Both groups of children became more efficient over the series, $F(3,84)=10.252, p<.001, \eta_{p}{ }^{2}=.268$. There was no main effect for visual status. For the adults, there were no significant main effects for repeated measurement of series or for visual status.

The dimension of volume showed a main effect for series, $F(3,171)=8.759, p<.001, \eta_{p}{ }^{2}=.133$, but not for age or visual status. All four groups of participants became more efficient over the series.

For the dimension of texture, there was a main effect for series, $F(3,171)=22.314, p<.001, \eta_{p}{ }^{2}=.281$, and a significant three-way interaction for series, visual status, and age, $F(3,171)=4.431, p<.005, \eta_{p}{ }^{2}=.072$. There was also a between-subjects interaction for age and visual status, $F(1,57)=4.667, p<.035, \eta_{p}{ }^{2}=.076$. For the sake of clarity, we analyzed the data further for the two age groups separately. These analyses showed a repeated measurement effect for the group of children, $F(3,84)=11.179, p<.001$, $\eta_{p}{ }^{2}=.285$; both groups of children became more efficient over the series. The analysis of the adults also showed a main effect for series, $F(3,87)=15.334, p<.001, \eta_{p}{ }^{2}=.346$, and a significant interaction effect for visual status by the repeated measurement of series, $F(3,87)=4.879, p=.003$, $\eta_{p}{ }^{2}=.144$. Separate analyses for the adults showed only a repeated measurement effect of series for the blind adults, $F(3,45)=15.856, p<.001, \eta_{p}{ }^{2}=.514$.
Fig. 5 Percentage of time exploratory procedures were used with the dimension of volume for the four groups for series 1 (a) and series 4 (b). $\mathrm{CF}=$ contour following, $\mathrm{EN}=$ enclosure, $\mathrm{LM}=$ lateral motion, $\mathrm{UH}=$ unsupported holding, $\mathrm{PR}=$ pressure
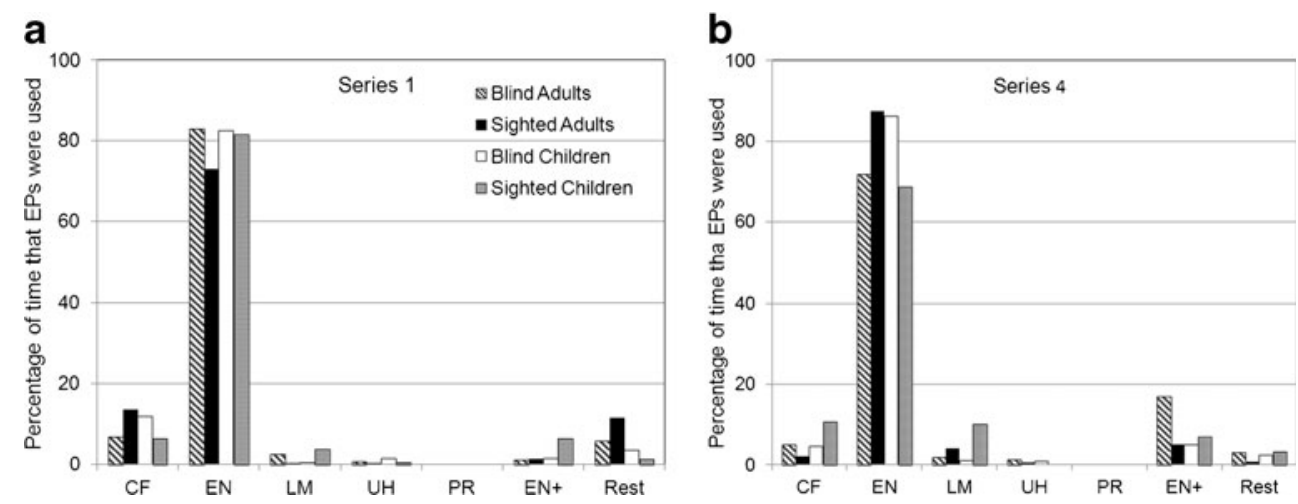
Fig. 6 Percentage of time exploratory procedures were used with the dimension of texture for the four groups for series 1 (a) and series 4 (b). $\mathrm{CF}=$ contour following, $\mathrm{EN}=$ enclosure, $\mathrm{LM}=$ lateral motion, $\mathrm{UH}=$ unsupported holding, $\mathrm{PR}=$ pressure a

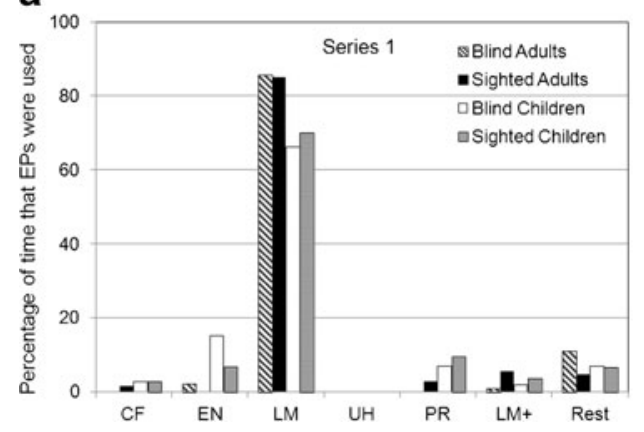

b

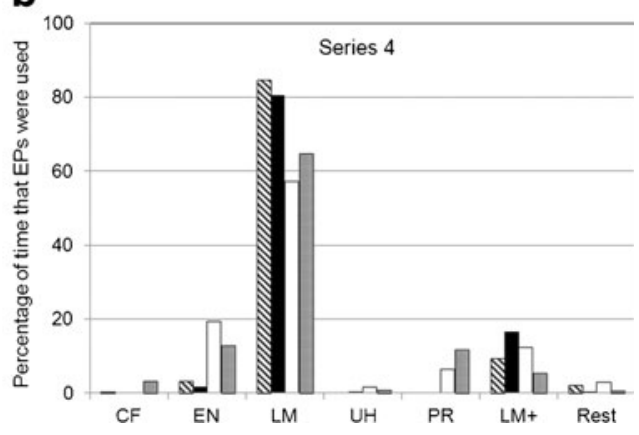

\section{Discussion}

The present study was an elaboration of our previous study in which we examined accuracy and response times of blind and sighted adults and children in a haptic match-to-sample task (Withagen et al., 2012). In that study, adults were found to be more accurate on all four dimensions but not always faster than children. In the present study, we looked at the EPs and the participants' efficiency during performance of the haptic match-to-sample task. All four groups of participants used the same EP to solve a specific task, in agreement with the study of Lederman and Klatzky (1987). However, the children also executed other EPs for the same dimension. Despite the extra hand movements, both groups of children became more efficient over four series on all four dimensions. The adults increased significantly in efficiency only on the dimensions of volume and texture. Below, we will discuss the three aims of the study separately-namely, differences in exploratory behavior of children and adults, differences in the manual exploration of blind and sighted participants, and the influence of practice - that is, repeating the experiment.
Exploratory behavior of children and adults

A similarity in the behavior of children and adults is the fact that they executed the same EP when information was asked about a specific dimension. The finding that children are able to execute the same EPs as adults is in line with the results of Kalagher and Jones (2011), who concluded that children produced adult-like EPs and matched the sample objects correctly to the standard on the different dimensions. Moreover, we found that both children and adults used some additional EPs, sometimes alone and sometimes in combination with the dominant EP. Noticeable was the fact that within a combination of EPs, all groups generally chose a combination that involved the dominant EP for that dimension. The fact that EPs could be performed simultaneously is in line with Klatzky and Lederman (1993) and Klatzky, Lederman, and Reed $(1989,1993)$, but in contradiction to Hatwell (2003), who stated that EPs cannot be executed simultaneously but must be performed successively, because they are not compatible from a motoric viewpoint. The simultaneous use of EPs, as predicted by Klatzky and Lederman (1993), did not occur very often during this experiment, but still it did.
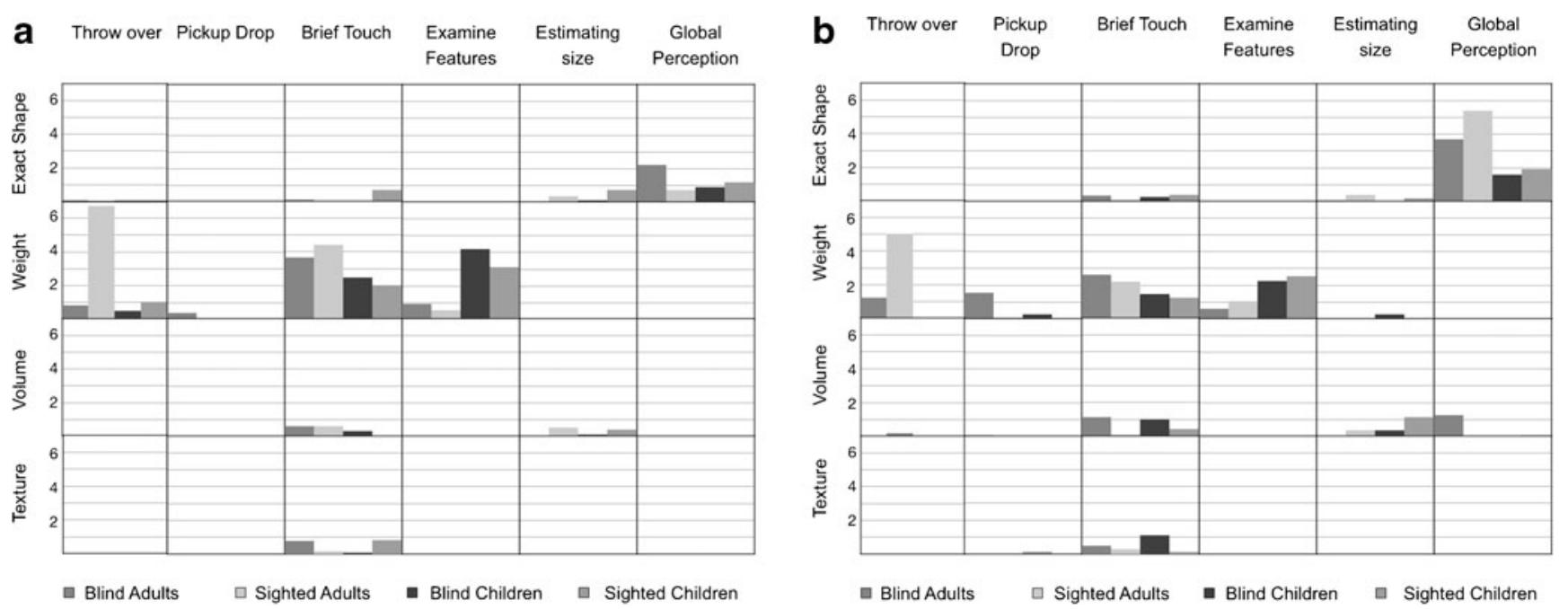

Fig. 7 Mean number of actions for series 1 (a) and series 4 (b) with each dimension for the four groups of participants 
Table 3 Mean duration in seconds of exploratory procedures for each object per trial. Data from the study of Lederman and Klatzky (1987) with students (L\&K students, $n=11$ ) and the four groups of participants in the present study

\begin{tabular}{|c|c|c|c|c|c|c|c|}
\hline & LM & PR & $\mathrm{SC}$ & $\mathrm{UH}$ & EN & $\mathrm{CF}$ & EPs total \\
\hline & \multicolumn{7}{|c|}{ Exact shape } \\
\hline L\&K students* & 0.35 & 0.00 & 0.00 & 0.03 & 1.92 & 11.20 & 13.50 \\
\hline Sighted adults & 0.00 & 0.00 & 0.00 & 0.00 & 0.20 & 13.04 & 13.24 \\
\hline Blind adults & 0.00 & 0.00 & 0.00 & 0.01 & 0.34 & 5.69 & 6.03 \\
\hline Sighted children & 0.10 & 0.00 & 0.00 & 0.00 & 0.40 & 5.31 & 5.81 \\
\hline \multirow[t]{2}{*}{ Blind children } & 0.04 & 0.00 & 0.00 & 0.01 & 0.47 & 5.31 & 5.82 \\
\hline & \multicolumn{7}{|c|}{ Weight } \\
\hline L\&K students* & 0.10 & 0.08 & 0.00 & 2.12 & 0.28 & 0.55 & 3.13 \\
\hline Sighted adults & 0.07 & 0.04 & 0.00 & 2.75 & 0.19 & 0.03 & 3.07 \\
\hline Blind adults & 0.08 & 0.00 & 0.00 & 2.87 & 0.13 & 0.00 & 3.07 \\
\hline Sighted children & 0.23 & 0.36 & 0.00 & 2.22 & 1.10 & 0.01 & 3.92 \\
\hline \multirow[t]{2}{*}{ Blind children } & 0.36 & 0.32 & 0.00 & 3.83 & 1.15 & 0.07 & 5.72 \\
\hline & \multicolumn{7}{|c|}{ Volume } \\
\hline L\&K students* & 0.20 & 0.01 & 0.04 & 0.07 & 2.61 & 2.15 & 5.08 \\
\hline Sighted adults & 0.06 & 0.00 & 0.00 & 0.07 & 2.79 & 0.59 & 3.51 \\
\hline Blind adults & 0.04 & 0.00 & 0.00 & 0.04 & 3.20 & 0.18 & 3.46 \\
\hline Sighted children & 0.05 & 0.00 & 0.00 & 0.01 & 2.92 & 0.72 & 3.69 \\
\hline Blind children & 0.02 & 0.00 & 0.00 & 0.10 & 3.18 & 0.73 & 4.02 \\
\hline EPs & \multicolumn{7}{|c|}{ Texture } \\
\hline L\&K students* & 3.46 & 0.18 & 0.00 & 0.00 & 0.07 & 0.82 & 4.53 \\
\hline Sighted adults & 3.11 & 0.22 & 0.00 & 0.00 & 0.00 & 0.06 & 3.38 \\
\hline Blind adults & 2.71 & 0.03 & 0.00 & 0.00 & 0.07 & 0.00 & 2.81 \\
\hline Sighted children & 2.71 & 0.54 & 0.00 & 0.00 & 0.38 & 0.12 & 3.75 \\
\hline Blind children & 2.79 & 0.41 & 0.00 & 0.00 & 0.74 & 0.15 & 4.10 \\
\hline
\end{tabular}

*Data based on Table 4a of Lederman and Klatzky (1987, p. 354)+

The percentage of time that children and adults spent on executing the dominant EP was found to vary substantially. Some children resembled adults in their exploratory behavior, while others used a lot of different EPs and manipulations, which could not be scored as the EPs described by Lederman and Klatzky (1987).This occurred especially on the dimensions of weight and texture. These manipulations involved motions to maintain an object in a certain position or to reorient it for further examination (scored as TM) or unclear motions that could not be interpreted as EPs or were visually UC. According to Millar (1994), children need to collect more redundant information about objects for proper use or identification of the objects, and for this reason, they will show more exploratory behavior. The extra hand movements are also understandable from a developmental perspective. To learn about the different dimensions of objects, children have to experiment more with objects than do adults because they lack experience with the different dimensions (Flavell, 1977; E. J. Gibson, 1988; J. J. Gibson, 1966; Hatwell, Orliaguet, \& Brouty, 1990; Shaffer, 2002). Therefore, one could expect that the children used more actions, since this is also a way to gather some extra information about objects. However, the results of the present study did not show more use of actions by the children, in comparison with the adults.

The actions should be interpreted as extra hand movements a participant executes to obtain more specific information about object properties or the stimulus itself. Certain actions concern sequences of hand movements in which EPs are involved, such as throw over, which starts with the EP of UH, followed by the action of throwing the object into the other hand and then, again, the EP of UH. To score this total range of hand movements as an "action" gives extra insight into the additional hand movements participants performed when exploring objects. A more stimulus-driven action is examine features, since the object itself (for instance, a hole in an object or a round object that can roll) evoked this behavior. Although provoked by different kinds of objects not used in the present study, the EP of function testing, described by Lederman and Klatzky (1987, p. 347) resembles this action. An action observed in all four groups and in all four tasks was brief touch. This action can be seen as a "quick glance" to extract information from an object (Klatzky \& Lederman, 1995). On the dimension of weight, the adults also 
Table 4 Mean scores per group for accuracy, response times, and efficiency scores

\begin{tabular}{|c|c|c|c|c|c|c|}
\hline & \multicolumn{2}{|c|}{ Accuracy in \% } & \multicolumn{2}{|c|}{ Response time in $\mathrm{s}$} & \multicolumn{2}{|c|}{ Efficiency $^{*}$} \\
\hline & Series 1 & Series 4 & Series 1 & Series 4 & Series 1 & Series 4 \\
\hline \multicolumn{7}{|l|}{ Exact shape } \\
\hline Blind adults & 86 & 89 & 102 & 77 & 1.08 & 1.37 \\
\hline Sighted adults & 87 & 88 & 152 & 133 & 0.64 & 0.76 \\
\hline Blind children & 70 & 63 & 93 & 60 & 0.83 & 1.21 \\
\hline Sighted children & 63 & 63 & 97 & 70 & 0.71 & 0.91 \\
\hline \multicolumn{7}{|l|}{ Weight } \\
\hline Blind adults & 84 & 88 & 56 & 52 & 1.70 & 1.90 \\
\hline Sighted adults & 82 & 83 & 59 & 64 & 1.65 & 1.42 \\
\hline Blind children & 63 & 73 & 93 & 61 & 0.76 & 1.34 \\
\hline Sighted children & 65 & 78 & 65 & 47 & 1.10 & 1.76 \\
\hline \multicolumn{7}{|l|}{ Volume } \\
\hline Blind adults & 86 & 94 & 59 & 47 & 1.69 & 2.43 \\
\hline Sighted adults & 93 & 97 & 60 & 50 & 1.79 & 2.09 \\
\hline Blind children & 88 & 85 & 68 & 47 & 1.57 & 2.21 \\
\hline Sighted children & 82 & 75 & 63 & 46 & 1.75 & 1.85 \\
\hline \multicolumn{7}{|l|}{ Texture } \\
\hline Blind adults & 94 & 100 & 49 & 33 & 2.31 & 3.51 \\
\hline Sighted adults & 97 & 100 & 53 & 48 & 2.11 & 2.40 \\
\hline Blind children & 93 & 93 & 69 & 44 & 1.47 & 2.41 \\
\hline Sighted children & 85 & 93 & 58 & 38 & 1.60 & 3.51 \\
\hline
\end{tabular}

* Note that, of course, efficiency averaged over individual participants does not equal the ratio of average accuracy and average response time

executed other actions, such as throw over and pick up and drop, whereas children rarely produced these actions. One can think of several reasons to explain this difference. Since, from a physiological viewpoint, the full maturation of the hands and its functions takes about 2 decades (Jones \& Lederman, 2006) and involves fine motor movements and the coordination of both hands (Hatwell et al., 1990). Throw over, as well as pick up and drop, might be a more mature motor and perceptual strategy to learn about the weight of objects. Moreover, the relatively small size of children's hands makes throwing over a difficult manipulation. Besides that, it might be necessary to practice or to learn about these actions by direct instruction or imitation before children are able to perform them well.

There was one action that was performed more by children than by adults, and that was examine features, but only on the dimension of weight. One could speculate that special features are affordances that evoke exploratory and playful behaviors in children more than they do in adults. Another explanation is that children in general perform more irrelevant behaviors than do adults, since Hatwell et al. (1990) observed that young children tend to include nonpertinent cues in their haptic explorations.
Exploratory behaviors of sighted and blind people

The blind and sighted participants resembled each other in the choice of EPs and actions on the four different dimensions. This means that in the absence of sight, blind people develop the same EPs to explore specific object properties as sighted people. The presence of visual abilities is apparently not a necessary condition for developing and conducting specific EPs. Moreover, both groups of children already use adult-like EPs to explore tactile dimensions. It seems that exploratory strategies depend more on the instruction than on whether a person is blind or sighted. This is in accordance with the conclusion of Lederman and Klatzky (1987) that specific EPs are mostly necessary (and not just optimal) to perform a certain task. The difference in behavior between blind and sighted participants lies in the efficiency of the execution of some hand movements. Especially, the congenitally blind adults were significantly more efficient, in comparison with the sighted adults, on the dimensions of exact shape and texture. In the execution of these specific haptic tasks, congenitally blind adults might profit from the fact that because of the lack of visual experience, they always carried out these tasks tactually. On the dimension of exact shape, the blind adults outperformed the sighted; they were more efficient, mostly because they used 
less time to execute EPs to achieve the same accuracy as the sighted adults. This might be explained by the advanced use of haptic strategies in which more fingers are involved in producing the EP of CF (Rovira et al., 2011). It is also the intensive usage of touch that makes it possible to develop special haptic competencies (Heller, 2000). Another possibility is that touch experiences may contribute to a change in brain activity, as was already mentioned by Vygotsky (1993), and higher efficiency in haptic shape recognition by blind adults, in comparison with sighted adults. Another explanation for the more efficient execution of the exact shape task by the blind could be the enhanced spatial attention mechanisms in blind people (Feindsod, Bach-y-Rita, \& Madey, 1973; Röder, Rösler, \& Henninghausen, 1997; Röder, Rösler, Henninghausen, \& Näcker, 1996). Research confirmed that blind persons need shorter discrimination times on spatial tasks due to these mechanisms. Within the group of children, we did not find this difference in efficiency, which could suggest that this attention mechanism and efficient use of fingers develop over time.

For children, there was a significant difference in performance between the two visual status groups on the dimension of weight. The sighted children were more efficient on this dimension than the blind; this is due to the longer response times of the blind children, as compared with the sighted children, especially in series 1 . An explanation could be that most blind children are explicitly taught to identify objects first. In the present experiment, they had to ignore this prominent feature. However, the results showed that the advantage of the sighted persons, in comparison with the blind, on the dimension of weight seems to disappear over time, since within the group of adults, we did not find a significant difference in efficiency.

\section{Influence of practice during the trials}

With practice, there was a decrease in the rest category of EPs in series 4. On all dimensions, there was a slight increase in the use of combined EPs and the use of an EP other than the dominant. Hand movements scored in the category of rest diminished over the series, and in series 4, $95 \%$ or more of the time EPs were carried out. Especially, on the dimension of weight, there was a large decrease in the category of rest (on average, from almost $20 \%$ to $5 \%$ ). The increased use of the dominant EP caused a significant improvement in efficiency for all four groups between series 1 and 4 for the dimensions of volume, exact shape, and texture. The children, but not the adults, also improved their performance on weight. Possibly, during the fourth series, they were less distracted by all the new affordances of an object (J. J. Gibson, 1966; Hatwell, 2003; Warren, 1994), which they could not ignore in the first series. Furthermore, practice might have led to more redundant information (Millar, 1994) and, as a result, also led to a more efficient exploration.

\section{Conclusion}

All four groups of participants executed the same dominant EP to gather information about a certain object dimension. The choice of the EP was determined by the sensory information they were searching for. Lederman and Klatzky (1987) already stated that task instruction immediately influences the way of exploring. All groups improved their exploratory behavior during practice. In general, the differences in exploratory behavior were affected more by age than by visual status. Only for the dimension of exact shape were there differences in the efficiency of the hand movements between sighted and blind adults, to the advantage of the latter group. This might be explained by the haptic experience and more advanced EPs in shape perception by the blind participants (Hatwell, 2003; Heller, 2000; Rovira et al., 2011). Between the two groups of children, there was a significant difference in performance only on the dimension of weight. However, the present study can be seen only as a first step in uncovering the variation in exploration behavior in blind and sighted participants at different age levels. To make a next step, it is important to conduct follow-up in-depth observational studies to further examine the individual variation in the execution of exploration tasks as a function of age and visual status.

Acknowledgments We are greatly indebted to all the participants, parents, and teachers. We also thank the students who observed the tapes and scored the response times. Leanne Looijens from the company Noldus was a great help to us with the analysis of the data of the EPs. Special thanks go to Dr. Lederman, who gave her permission to reproduce the stimuli of the original object set and cooperated in realizing this set. We also render thanks to Dr. Lederman and Dr. Klatzky for their comments on some preliminary results. The research was supported by a grant from the Novum Foundation, a nonprofit organization providing financial support to (research) projects that improve the quality of life of individuals with a visual impairment. A. M. L. Kappers was supported by a grant from the EU project no. 248587, "The Hand Embodied."

\section{References}

Berger, C., \& Hatwell, Y. (1993). Dimensional and overall similarity classifications in haptics: A developmental study. Cognitive Development, 8, 495-516.

Berger, C., \& Hatwell, Y. (1995). Development of dimensional vs. global processing in haptics: The perceptual and decisional determinants of classification skills. British Journal of Developmental Psychology, 13, 143-162.

Berger, C., \& Hatwell, Y. (1996). Developmental trends in haptic and visual free Classifications: Influence of stimulus structure and exploration on decisional Processes. Journal of Experimental Child Psychology, 63, 447-465.

Bushnell, E. W., \& Boudreau, J. P. (1991). The development of haptic perception during infancy. In M. A. Heller \& W. Schiff (Eds.), The psychology of touch (pp. 139-161). Hillsdale, New Jersey: Lawrence Erlbaum Associates. 
Cornoldi, C., \& Vecchi, T. (2000). Mental imagery in blind people: the role of passive and active visuospatial processes. In M. A. Heller (Ed.), Touch, Representation, and Blindness (pp. 143-183). Oxford: University Press.

Cornoldi, C., Fastame, M., \& Vecchi, T. (2003). Congenitally blindness and spatial mental magery. In Y. Hatwell, A. Streri, \& E. Gentaz (Eds.), Touching for knowing (pp. 173-187). Amsterdam: John Benjamins Publishing Co.

Davidson, P. W. (1972). Haptic judgements of curvature by blind and sighted humans. Journal of Experimental Psychology, 93, 43-55.

Davidson, P. W., \& Whitson, T. T. (1974). Haptic equivalence matching of curvature by blind and sighted humans. Journal of Experimental Psychology, 102, 687-690.

Feindsod, M., Bach-y-Rita, P., \& Madey, J. M. (1973). Somatosensory evoked responses: Latency differences in blind and sighted persons. Brain Research, 60, 219-223.

Flavell, H. F. (1977). Cognitive development. New Jersey: PrenticeHall.

Gibson, J. J. (1966). The senses considered as perceptual systems. Boston: Houghton-Mifflin.

Gibson, E. J. (1988). Exploratory behavior in the development of perceiving, acting, and acquiring of knowledge. Annual Review of Psychology, 39, 1-41.

Goldreich, D., \& Kanics, I. (2003). Tactile acuity is enhanced in Blindness. The Journal of Neuroscience, 23(8), 3439-3445.

Grant, A. C., Thiagarajah, M. C., \& Sathian, L. (2000). Tactile perception in blind Braille readers: A psychophysical study of acuity and hyperacuity using gratings and dot patterns. Perception \& Psychophysics, 62, 301-312.

Hatwell, Y. (2003). Manual exploratory procedures in children and adults. In Y.Hatwell, A.Streri \& E.Gentaz (Eds.), Touching for knowing (pp. 67-83). Amsterdam: John Benjamins Publishing Co.

Hatwell, Y., Orliaguet, J. P., \& Brouty, G. (1990). Effects of object properties, attentional constraints and manual exploraotry procedures on haptic perceptual organization: a developmental study. In H. Bloch (Ed.), Sensory-motor organizations and development in infancy and early childhood (pp. 315-335). Dordrecht: Kluwer Academic Publishers.

Heller, M. A. (1989). Texture perception in sighted and blind observers. Perception \& Pscyhophysics, 45(1), 49-54.

Heller, M. A. (2000). Conclusions: the San Marino discussion. In M. A. Heller (Ed.), Touch, Representation, and Blindness (pp. 183214). Oxford: University Press.

Jones, L. A., \& Lederman, S. J. (2006). Hand function across the lifespan. In L. A. Jones \& S. J. Lederman (Eds.), Human Hand Function (pp. 150-178). Oxford: University Press.

Kalagher, H., \& Jones, S. (2011). Young children's haptic exploratory procedures. Journal of Experimental Child Psychology, 110, 592-602.

Klatzky, R. L., Lederman, S. J., \& Reed, C. (1989). Haptic integration of Object Properties: Texture, Hardness and Planar Contour. Journal of Experimental Psychology: Human Perception and Performance, 13, 45-57.

Klatzky, R. L., Lederman, S. J., \& Reed, C. (1993). Constraints on haptic integration of spatially shared object dimensions. Perception, 22, 723-743.

Klatzky, R. L., \& Lederman, S. J. (1993). Towards a computational model of constraint-driven exploration and haptic object identification. Perception, 22, 597-621.
Klatzky, R. L., \& Lederman, S. J. (1995). Identifying objects from a haptic glance. Perception \& Psychophysics, 57(8), 1111-1123.

Klatzky, R. L., Lederman, S. J., \& Mankinen, J. M. (2005). Visual and haptic exploratory. procedures in children's judgements about tool function. Infant Behavior \& Development, 28, 240-249.

Landau, B. (1991). Spatial representation of objects in the young blind child. Cognition, 38, 145-178.

Lederman, S. J., \& Klatzky, R. L. (1987). Hand movements: a window into haptic object recognition. Cognitive Psychology, 19, 342-368.

Millar, S. (1974). Tactile short-term memory by blind and sighted children. British Journalof Psychology, 65, 253-263.

Millar, S. (1994). A theory of spatial understanding and development. In S. Millar Understanding and representing space. Oxford: Clarendon Press.

Millar, S. (1997). Theory, experiment and practical application in research on visual impairment. European Journal of Psychology of Education, 12(4), 415-430.

Millar, S. (2008). Space and Sense. New York: Psychology Press.

Morange-Majoux, F., Cougnot, P., \& Bloch, H. (1997). Hand tactual exploration of textures in infants from 4 to 6 months. Early Development \& Parenting, 6, 127-135.

Noldus Information Technology (2008). The Observer XT Reference Manual Version 8.

Röder, B., Rösler, F., Hennighausen, E., \& Näcker, F. (1996). Eventrelated potentials during auditory and somatosensory discrimination in sighted and blind human subjects. Brain Research, Cognitive Brain Research, 4, 77-93.

Röder, B., Rösler, F., \& Hennighausen, E. (1997). Different cortical activiation patterns in Blind and sighted humans during encoding and transformation of haptic images. Psychophysiology, 34, 292307.

Rovira, K., Deschamps, L., \& Baena-Gomez, D. (2011). Mental rotation in blind and sighted adolescents: The effect of haptic strategies. Revue Européenne de psychologie appliqué, 61, 153-160.

Shaffer, D. R. (2002). Developmental psychology: Childhood and adolescence. Belmont, CA: Wadsworth/Thomson Learning.

Schellingerhout, R., Smitsman, A. W., \& Van Galen, G. P. (1997). Exploration of surface-textures in congenitally blind infants. Child: Care, Health and Development, 23(3), 247-364.

Schwarzer, G., Küfer, I., \& Wilkening, F. (1999). Learning categories by touch: On the development of holistic and analytic processing. Memory \& Cognition, 27, 868-877.

Vanlierde, A., \& Wanet-Defalque, M. (2004). Abilities and strategies of blind and sighted subjects in visuo-spatial imagery. Acta Psychologica, 116, 205-222.

Vecchi, T., Tinit, C., \& Cornoldi, C. (2004). Spatial memory and integration processes in congenital blindness. Cognitive neuroscience and neuropsychology, 15, 2787-2790.

Vygotsky, L. S. (1993). The blind child. In R. W. Rieber \& A. S. Carton (Eds.), The collected Works of L.S. Vygotsky, Volume 2 The fundaments of Defectology (pp. 97-109). New York: Plenum Press.

Warren, D. (1994). Executive functions: memory, attention, and cognitive strategies. In D. Warren \& Blindness and children (Eds.), An individual differences approach. New York: Cambridge: University Press.

Withagen, A., Kappers, A. M. L., Vervloed, M. P. J., Knoors, H., \& Verhoeven, L. (2012). Haptic object matching by blind and sighted adults and children. Acta Psychologica, 139, 261-271. 